\title{
A Review of Tools Applied in Processing of Medical Images
}

\author{
${ }^{1}$ Anandakumar Haldorai and ${ }^{2}$ Shrinand Anandakumar \\ ${ }^{1}$ Computer Science and Engineering, Sri Eshwar College of Engineering, India. \\ ${ }^{2}$ PSBB Millennium School, India. \\ 1anandakumar.psgtech@gmail.com
}

\author{
Article Info \\ Journal of Biomedical and Sustainable Healthcare Applications (http://anapub.co.ke/journals/jbsha/jbsha.html) \\ Doi: https://doi.org/10.53759/0088/JBSHA202101006
}

Received 28 August 2020; Revised form 25 September 2020; Accepted 28 October 2020.

Available online 05 January 2021.

(C2021 Published by AnaPub Publications.

\begin{abstract}
The segmentation step of therapy treatment includes a detailed examination of medical imaging. In diagnosis, clinical research, and patient management, medical pictures are mainly utilized as radiographic methods. Image processing software for medical imaging is also crucial. It is possible to improve and speed up the analysis of a medical picture using a bioMIP technique. This article presents a biomedical imaging software tool that aims to provide a similar level of programmability while investigating pipelined processor solutions. These tools mimic entire systems made up of many of the recommended processing segment within the setups categorized by the schematic framework. In this paper, 15 biomedical imaging technologies will be evaluated on a number of different levels. The comparison's primary goal is to collect and analyze data in order to suggest which medical image program should be used when analyzing various kinds of imaging to users of various operating systems. The article included a result table that was reviewed.
\end{abstract}

Keywords - Neural Edge Enhancer (NEE), Medical Image Processing (MIP), Signal processing (SP)

\section{INTRODUCTION}

Information Technology (IT) has infiltrated every area of human existence, and the effect of this development on the disciplines of health and medical research has been evident in recent years. Medical science is based on biomedical imagery. Pictures of the body (that aid in the knowledge of the aspects of the human natural ecosystem) are referred to as these images. Any method established for one dimension may be extended to columns and then rows in this digital picture, which is represented as a matrix of columns and rows. These medical images include whole organs, body functions, and bodily sections, as well as molecular structures. The methods of image processing are very important in comprehending and collecting information from these pictures. Medical imaging is the process of providing a better understanding of the inside sections or interior portion sizes of a body for diagnostic purposes, surgical treatment, and graphical demonstration of the component of a few anatomical structures for diagnostic purposes, medicative intrusion, and graphical demonstration of the component of a few anatomical structures. With the enhanced communication connections and policies and procedures, such as Picture Archiving and Communication Systems (PACS), Interactive Image analysis and Communication services in Medical science, these visual manifestations are proficiently processed, analyzed with strategic targets, and released publicly and communicated to multiple locations [1]. With the assistance of computerized image processing methods in the medical sector, doctors have been able to quickly identify illnesses.

For clinical diagnosis, a variety of methods and instruments are available. The uses of the stochastic environment for clinical imaging are discussed in depth in [2]. Therapeutic and diagnostic healthcare systems, spanning from medical standards to micro-implants, common screening equipment like MRIs and EEGs, regeneration granulation tissue, pharmacological medicines, and medicinal biology are all examples of biomedical engineering fields. Nanotechnology is a relatively young field of science that investigates instruments and systems with different purposes at the biological, subatomic, and cellular scale, ranging in size from 1 to 100 nanometers. With the creation of novel microparticles for the monitoring and diagnosis of cancer, there has been a lot of progress in the area of nanotechnology in recent times. Nanoparticles possess biological characteristics due to their tiny size and great surface - to - volume ratio, which enables them to efficiently bind, absorb, and transport substances such small molecule medicines, DNA, RNA, peptides, and probe.

The two types of picture pretreatment using neural network models are image reconstructions and image enhancement. One of the most applied neural networking framework for the restoration of images is the Sequence - to - sequence multilayer perceptron. In these domains, Hopfield network-based techniques account for 55 percent of the publications we looked at. The primary benefit of employing Hopfield neural network frameworks for diagnostic restoration of images is that the issue can be seen as an optimal process that can be readily addressed by allowing the networks to convergence to a steady position while reducing the energy component. The computation of a quadratic inversion on chaotic data is required for image restoration in amperometric radiography. This issue is often ill-conditioned, necessitating either assumptions simplification or batch normalization based on previous information. When compared to other methods, the feed forward 
neural network as well as the self-organizing Convolution neural networks, which each have two articles in our examined literature, seem to have greater benefits. From numerical simulation models of the forward issue, they can immediately compute a linear function of the inverse problem. Medical image enhancement is where the bulk of artificial neural applications in medicine picture preprocessing may be found. For this issue, Suzuki and colleagues created neuronal Network Filters (NFs).

K. Suzuki, I. Horiba and N. Sugie in [3] have developed a novel Neural Edge Enhancer (NEE) for clearly boosting the required edges from noisy pictures, which is based on a customized multilayer perceptron. The NEE is an edge enhancer that requires supervision: The NEE learns the functionality of a specified edge enhancement via training using a collection of input noisy pictures and instructional edges. NEE outperformed traditional edge enhancers in terms of noise resistance, ability to improve uninterrupted endpoints from noisy pictures, and edge similarity. For medical picture categorization, the convolutional networks are the most used multilayer perceptron. The feed forward networks were used for medical picture segmentation in six of the 17 publications we examined. It is acknowledged that the feed forward neurological networking segmented photos are considered to be minimally fussy compared to the contemporary Maximum Likelihood Classifiers (MLC) image fragmentation methodology and that the classifiers of the feed forward neural network is minimally sensitive when it comes to the process of training a collection of selection compared to MLC. Majority of the feed forward neural networks and their methodologies have a sluggish rate of convergence and necessitate the integration of the parameters of learning that are identified overhead. The feed forward neural network's application within the clinical photography segmentations is limited due to a number of demerits. The Hopfield neural networks have been considered as a methodology for mitigating the challenging optimization issues, which had previously been difficult to mitigate. This renders them a more applicable alternative to conventional optimization approach for clinical photograph construction that might be illustrated as an issue. 4 of the journal that we evaluate utilized the Hopfield neural network to effective illustrate a separation of organs from a clinical photograph.

For medical image detection and verification, the backpropagation networks is the most often employed multilayer perceptron [4]. It appeared in 11 publications we examined for this review. All of these feed-forward neuron based $\mathrm{m}$ optimization techniques outperform customary image recognition methodologies, whether they are used for mammography explanation, cold lesion identification in SPECT visuals, diagnosing courses of liver diseases based on ultrasound imaging, differentiating melanoma from 3 benign classifications of tumor cells, or lowering false positive results in computer controlled diagnosis of pulmonary nodules in LDCT and chest radiation therapy. Other artificial neural network, like the Hopfield network, the ART classification techniques, the radial basis function network, the Stochastic Multilayer Perceptron, the convolutionary neural connection, and the recursive neural system, have also found a spot in brain tumor detection classification and tracking, with 23 publications.

In contrast to what was previously claimed, convolutional neural network groupings are utilized for cancer detection in [5]. There are two layers to the ensemble. When every individual network only has multiple outcomes, conventional cell or unicellular organism, the first-level ensemble is utilized to effectively illustrate the normal aspect of the cell. To incorporate the projections of different networking models, an approach is applied. The second-level ensembles is applied to cope with the cell identified as a cancer cell by the ensemble in the first level with each neural networks having many outputs, each signifying a varied form of pulmonary cancerous cell. The forecasts of the various networks are integrated utilizing the commonly applied approach known plurality voting. The experiment illustrated that the neural networking ensemble is capable of attaining not only a higher percentage of overall recognition, but also a minimal interest rate of the false negative identifiers (that is a lower rate of assessing cancerous cells as the human cell that is fundamental in minimizing cancer victim diagnosis issues and saving patients' lives).

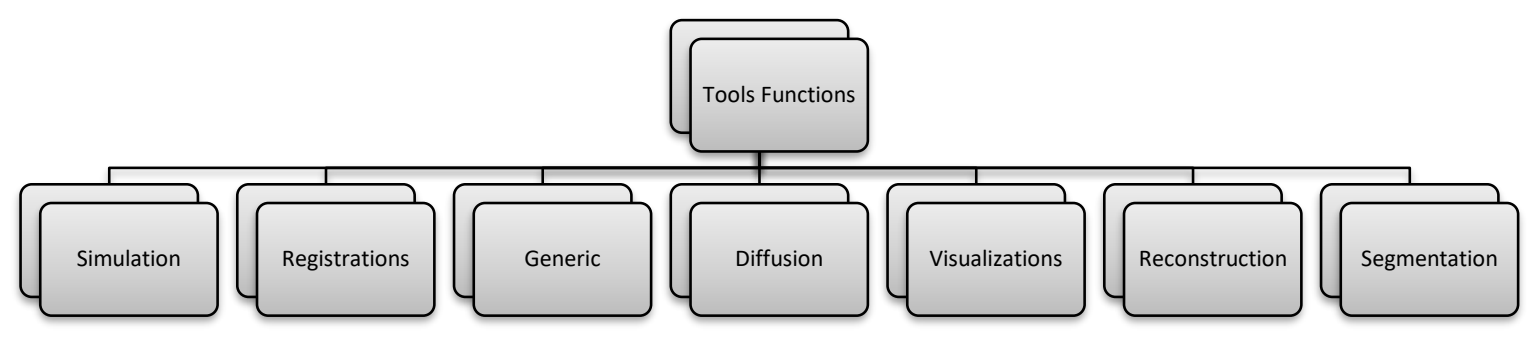

Fig 1. Tools' Function

Image segmentation is a kind of data processing that uses pictures as the output and input, e.g. videos and photos sequences. Images are often processed as 2D impulses and then subjected to conventional SP methods. Digital image analysis and medical image treatment are the two types of image processing that may be found in general [6]. Medical Image Processing (MIP) technologies will be the subject of this article. Interventional radiology and analytics technologies are becoming important in a variety of applications in the medical sector. Such applications may be found all along the clinical timeline, not only in medical area, but also in the domains of surgical preparation, execution, and assessment. As a consequence, the advantages and disadvantages of the ultrasound images will have a direct impact on the outcome of the doctor-patient diagnostic. Furthermore, medical imaging, like ultrasound, has noise and speckle, making physicians' decisions more challenging. The purpose of this article is to collect and evaluate a wide range of medial image analysis 
technologies currently available. To determine which tools are free software and which tools are compatible with which operating systems, see the table below. Many image analysis tools have been developed to perform various tasks, such as diffusion, simulation, reconstruction, visualization, segmentation, registration as shown in Fig 1.

\section{FOUNDATION}

This section will offer a short orientation to medical image analysis technologies and machine vision as a basis for the subsequent survey and debate. This section will also cover the basic operations of a biomedical image analysis tool.

\section{Image Editing Software}

The look of a picture is one of the main goals of image processing. There are many image processing programs available. Image Processing Tools are a collection of plugins, development tools, algorithms, and applications for image processing methods that scientists and researchers may use. The majority of visual processing methods consider the picture as a twodimensional signal that is then processed using conventional pattern recognition techniques. The kind of image processing is shown in the diagram below. Imaging processing techniques tools and therapeutic image analysis tools are the two major kinds of image processing technologies.

\section{Tools for Image Processing Technique}

Digital image analysis, as per T. Cevik, M. Fettahoglu, N. Cevik and S. Yilmaz in [7], is indeed the technique of doing image analysis on digital pictures using computer algorithms. Smart image processing, as the subset or segment of the digitalized framework, provides various benefits over the analogue image evaluation. It enables a considerably broader variety of techniques to be employed to the data input, as well as the avoidance of issues like noise accumulation and signal deformation throughout processing. Many of the methods used in image processing technique, also known as digital image representation, were created in the mid-1960s.

\section{Tools for MIP}

The method, technique, and art of generating visualisations of the inside of an organism for pathological evaluation and surgical treatment is referred to as computed tomography. Tomography aims to show hidden interior structures under the bones and skin, and detect and cure illness. The aspect of tomography is develops a catalog of typical anatomy and physiology, permitting anomalies to be identified. Even though clinical imaging is excised tissues and organs, these operations are categorized as pathology instead of clinical imaging form. As a result, the emphasis of this article will be on a review of therapeutic image processing technologies. A brief review of several kinds of image analysis tools will be presented in the following section.. The biomedical imaging technologies that will be discussed in section III are shown in the diagram below.

\section{Medical Illustrations}

Medical imaging has advanced significantly in the contemporary medical sector. This technique is significant since it may be used prior to surgery. Various types of diagnostic imaging have indeed been established throughout time. Different kinds of medical pictures respond to various technologies. Each medical picture has its own set of benefits and drawbacks. Fig 2 depicts 15 different kinds of medical image analysis technologies available on the market.

Medical image enhancement may be done using a variety of software programs. The section that follows presents 15 different kinds of instruments that are often utilized by scientists.

\section{GIMIAS}

The Graphical Interface for Medical Image Analysis and Simulation (GIMIAS) represents the workplan model for focusing on the crucial clinical image customized and computing simulation issues that may be extended with problemspecific connector.

\section{NiftyReg}

NiftyReg is a free and open-source therapeutic picture registrations program. It was primarily created by researchers of the Therapeutic Radiology Group at University London's Centre for Tumor Detection Computing.

\section{Elastix}

Elastix is free software that is based on the popular Insight Integration and Registering Toolkit. The program is made up of a set of techniques that are often used to address (medicine) picture registration issues. Elastix's modular architecture enables users to easily setup, test, and compare several registrations mechanisms for a given application.

\section{ANTS}

B. Avants, N. Tustison, G. Song, P. Cook, A. Klein and J. Gee in [8] developed ANTS, or Advanced Standardization Tools. An ANT can extract data from large databases, making it ideal for organizing, analyzing, and displaying multimedia data. 
NiftySegment

NifySeg is among the University College London programs that are released under the License agreement. It's a collection of applications that do EM-based picture segmented in simple or analytical formats.

\section{ITK-Snap}

ITKSNAP supports semi-automated segmented using active contour techniques, and also manual demarcation and picture navigation.

\section{MITK}

The Radiology Interactive Framework represents an initiative and model, which incorporate the Insights Toolkit and VTK (Visual Toolkit). The application has been permitted under the BSD guideline.

NiftyRec

NiftyRec is a computed tomography reconstructions application project created by University College London.

NiftySim

University London created Nifty Sim, a high-performance non - linear finite component simulator. The possibility of GPU-based computation is a crucial feature, allowing the solver to substantially outperform comparable commercialized programs.

\section{Camino}

Camino is a computer framework for displacement MRI treatment that allows you to build processing pathways using components from other programs. The toolbox is now being maintained by the substructure photography group at UCL, which is in charge of developments.

\section{The DTI-TK}

DTI-TK is a toolbox for analyzing brain structure morphology employing DTI data that includes spatial standardization and atlas building. A study was published in the journal NeuroImage in 2011. DTI-TK was named the outstanding instrument in its category.

\section{$V T K$}

VTK (Visualization Toolkit) is a freeware, open-source computer application for 3D visualization, computer vision, and visual analytics. This function is evaluated by G. Wills in [10], whose group is still working on continued expansion of the toolkit. It also provides professional assistance and consultancy for VTK. VTK also includes comprehensive visual analytics architecture, a library of 3D interface elements, concurrent processing capabilities, and integration with database systems on GUI development tools like QT or Tk.

\section{ITK}

The Insight identification and fragmentation or ITK represents a cross-platform and open-sourced initiative, which provides programmers access to a wide range of image analysis tools. ITK also includes cutting-edge separation and identification techniques in 2,3, and much more domains. It's available as open-source software - defined data center.

FSL.

Systems Perspective, OXFORD, and FMRIB, UK collaborated to develop FSL (FMRIB Systems Library). FSL is a collection of technique for evaluating FMRI, MRI, and DTI electroencephalography data.

\section{$S P M$}

The development and evaluation of geographically extended measurement methods used to evaluate assumptions regarding functional image sequences is referred to as supervised classification mapping. SPM, a piece of software, has been created to implement these concepts. Karl Friston created the SPM software package to analyze electroencephalography sequence of data eg MEG, EEG, SPECT, PET, and Fmri. 
Clinical Image Processing Instruments



Fig 2. Medical Image Analysis Tools 


\section{DISCUSSION}

In this section, Table I tabulates the results of the evaluation of 15 biomedical imaging programs. The following parameters are used to analyze the data. A contrast of the 15 medical image analysis technologies mentioned is shown in the Table 1 below.

Table 1. Contrast of the 15 medical image analysis technologies

\begin{tabular}{|c|c|c|c|c|c|c|c|c|c|c|c|c|c|c|c|c|}
\hline \multicolumn{2}{|l|}{$\begin{array}{l}\text { Image } \\
\text { Processing } \\
\text { Instrument }\end{array}$} & $\begin{array}{l}\text { DTK } \\
\text { TK }\end{array}$ & CAMINO & $\begin{array}{l}\text { NIFTY } \\
\text { SIM }\end{array}$ & $\begin{array}{l}\text { NIFTY } \\
\text { REC }\end{array}$ & MITK & ITK & $\begin{array}{l}\text { NIFTY } \\
\text { SEG }\end{array}$ & ANTS & ELASTIC & $\begin{array}{l}\text { NIFTY } \\
\mathrm{REG}\end{array}$ & GIMIAS & SPM & FSL & ІтК & VTK \\
\hline \multirow[t]{8}{*}{ Function } & Diffusion & $*$ & $*$ & & $*$ & * & & & & $*$ & & * & $*$ & & & $*$ \\
\hline & Simulation & $*$ & & $*$ & $*$ & & $* *$ & & & $*$ & & $*$ & & $*$ & & $*$ \\
\hline & Reconstruction & $*$ & & $*$ & $*$ & & $*$ & & $*$ & * & & $*$ & $*$ & * & & $*$ \\
\hline & Visualization & & $*$ & $*$ & $*$ & $*$ & $*$ & & & $*$ & $*$ & $*$ & & & $*$ & \\
\hline & Segmentation & & $*$ & $*$ & $*$ & $*$ & & * & & $*$ & & $*$ & $*$ & & & $*$ \\
\hline & Registration & $*$ & $*$ & & & $*$ & & * & $*$ & $*$ & $*$ & $*$ & & 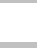 & $*$ & \\
\hline & Generic & & $*$ & & & & & & $*$ & $*$ & $*$ & & $*$ & $*$ & $*$ & $*$ \\
\hline & $\begin{array}{l}\text { Three- } \\
\text { dimensional } \\
\text { images }\end{array}$ & $*$ & & & $*$ & $*$ & $*$ & $*$ & & $*$ & & & & & & \\
\hline \multirow{7}{*}{$\begin{array}{l}\text { System } \\
\text { Languages }\end{array}$} & VB NET & & & & & & & & & * & & & & & & \\
\hline & Python & & & & $*$ & $*$ & & & $*$ & & & $*$ & & & & $*$ \\
\hline & Java & $*$ & * & & & & & & & & & & & & $*$ & $*$ \\
\hline & PHP & & & & $*$ & & & & & & & & & & & \\
\hline & $\mathrm{C}++$ & & & & & & & & & & $*$ & & $*$ & & & $*$ \\
\hline & $\mathrm{C}$ & & & & & $*$ & $*$ & & & & & & & $*$ & & \\
\hline & $\mathrm{C \#}$ & & & & & & & * & & & & & & & & \\
\hline \multicolumn{17}{|l|}{ Paid } \\
\hline \multicolumn{2}{|c|}{ Open Source } & $*$ & $*$ & * & $*$ & $*$ & * & * & $*$ & * & $*$ & * & * & $*$ & $*$ & * \\
\hline \multicolumn{2}{|l|}{ Framework } & & & & & & & & & & & $*$ & & & & \\
\hline \multicolumn{2}{|c|}{ Plugin/Integration } & $*$ & & $*$ & & & & * & & & & & & * & & $*$ \\
\hline \multicolumn{2}{|c|}{ Standalone Tools } & & $*$ & $*$ & $*$ & $*$ & $*$ & & $*$ & $*$ & $*$ & & $*$ & $*$ & $*$ & \\
\hline \multirow[t]{3}{*}{ Platforms } & Mac OS X & $*$ & $*$ & & $*$ & $*$ & $*$ & * & $*$ & $*$ & $*$ & & & & $*$ & $*$ \\
\hline & Linux & $*$ & $*$ & $*$ & $*$ & $*$ & $*$ & * & $*$ & $*$ & $*$ & $*$ & $*$ & $*$ & $*$ & $*$ \\
\hline & Windows & $*$ & $*$ & $*$ & $*$ & $*$ & $*$ & & $*$ & $*$ & $*$ & $*$ & $*$ & $*$ & $*$ & $*$ \\
\hline
\end{tabular}

As seen above, evaluations between 15 medical image analysis technologies have been collated. The newest version of instruments accepted, system GUI endorsed, tomography (modes) endorsed, tool functionalities offered, tool linguistics, tool kinds, pricing, and the category of candidate to launch on are the parameters for the evaluations to the consumers as usual. Based on GUI, the 15 sophisticated tools provide individuals with an interface control, making GUI displays userfriendly and easy for newcomers. Different supporting modalities are available for each of the fifteen medical image analysis technologies. VTK, for example, only offers 3D imaging techniques, while SPM can support five different forms of clinical imaging, integrating MITK, ELASTIX, GIMIAS, EEG, CT-SCAN, PET, FMRI, and MRI supporting the various forms of clinical photographs.

In addition, every tool has its own set of capabilities. NiftyReg, for instance, has the least functionality; it just allows fragmentation and visualisation, while Elastix and Camino include all of their features. The languages utilized to create the software package will be the next subject of debate. DTI TK, CAMINO, ITK SNAP and FSL, on the contrary represent the C\# tools. NiftSeg, MITK, and SPM are the methods within the C programs. Elastix, GIMIAS, and VTK represent the C++ application. Java intergrates DTI-TK, CAMINO and ITK. The tools' reminders are python-centered eg NiftSim, NiftyRec, NiftySeg, NiftyReg. ANTs alone have written the visual Basics.Net. NiftySeg, FSL, VTK provide the compatibility and extension with other IDE sequences e.g. Matlab. The MIP initiatives evaluated can operate as a standalone initiative, whereas VTK, NiftySeg and FSL provide interfaces and connectors with the respective IDE programs. GIMIAS is a standalone model. Most technologies fall under MIP.

Some of the apps examined offer a free version that may be used for non-commercial purposes, while others have trial version that last one to three months. Some MIP tools need individuals to purchase permits to unlock the complete operation functionalities of the MIP model. Various MIP instrument operate under different platforms such as Mac OS X Linux, and Windows and might be installed on various computers, which support Java. Programmers would recommend Elastix for MIP with respect to the users' experience and survey data since it is presently becoming a tool that provide the most comprehensive and friendly experience to the users. It allows clients to obtain a more recent version of the application's usage and it integrates a graphical interface for the users. In addition, it supports CT scans, X-rays, Ultrasound, and MRI among the wide-range imaging methodologies. Whenever conducting imaging evaluation, it provides a wider array of functionalities, and it might operate as a standalone model or linked with various IDEs such as Visual Studio, Netbean, and Matlab. Elastix is considered an open and free source program. 
Users may get the most recent version from the company's official website. Aside from that, the website offers beginning lessons. Elastix is an all-in-one MIP solution. It also offers a variety of options for importing and exporting data. Medical pictures may be saved in different formats such as TIFF, PNG, Bitmap, JPEG and Dicom. As a result, the Elastix MIP tool serves as a really comprehensive MIP tool, with a broad range of capabilities, file type imports and exports, and compatibility for a wide range of platforms. The Elastix for MIP tool is suggested for those seeking for a full-featured experience. Users who wish to test out a segmentation or visualization tool for the first time should utilize the Camino, which is the same as Elastix in supporting the various forms of clinical imaging and have the various elements mentioned. Nonetheless, it can be integrated within the windows operating system. Despite the fact that Camino lacks an installation like other operating systems, it is a decent fundamental functioning utility. In the table above, many MIP tools that are available for non-commercial application as considered as such. The webpages for different applications provide free download. The MIP tools, which are not indicated as free necessitate customers to buy a license in order to use them.

\section{THE FUTURE OF IMAGE PROCESSING}

In case you have ever gone to the ER for an injury, you have probably had a regular contact with what is, in reality, a modern-day marvel: Magnetic Resonance Imaging, or MRI. Medical imaging advances have enabled us to examine sections of the body, particularly the brain, to a level never before seen in medical research. But how are MRI pictures created in a non-invasive and radiation-free manner? They depend on thermodynamics and Signal processing (SP), in a nutshell. And, using medical imaging technologies signal conditioning engineers are creating innovative new methods to investigate the human brain and nervous system in a safe manner.

Magnetic fields used in MRI are 60000 times more powerful than those used in a typical refrigerator magnet. The protons of hydrogen atoms are targeted by this magnetic field, which causes each proton to align with or against the field. High frequency electrical pulses are injected repeatedly. When a pulse is activated, the protons are knocked out of synchronization, and when the pulse is shut off, the protons progressively realign. Thousands of radio signals are produced when the electromagnetic fields pulse is switched on and off again. This data is sent to various pixel positions by variations in the magnetism. The spatial and spectral elements of the MRI picture are formed by this output, which is referred to as the K-space. The importance of SP in this process cannot be overstated. On the K-space data, we use a popular SP technique known as the Inversion of the Fourier Transformation to create the MRI picture. The Fast Fourier Transform, or "FFT," detects the frequencies in a signal and is one of the most flexible tools for researchers and technologists [11-16].

\section{Overcoming Biomedical Imaging Techniques Constraints}

SP experts, on the other hand, are looking at methods to improve the picture building process. A perfect picture takes at least an hour or more in most MRIs. This has two negative consequences: it puts a burden on the healthcare facility's logistics (scanning a small number of patients) and it causes individual discomfort and claustrophobic. (Anyone who has ever waited inside an Ultrasound probe understands how aggravating that can be!) There is a requirement for a quicker response time. Certain wavelength K-space information is often deleted or shortened to speed up picture creation, with the Inverse Transform applied solely to the remaining data. However, this isn't the best option since it causes the field of vision to be narrower than the body portion being photographed (a process known as "aliasing artifacts"), as well as the Gibbs phenomena (a ringing caused by abbreviating the K-space harmonics).

So, how can we improve our pictures without having to wait much longer? Compressed Sensing is one of the methods being developed by SP experts to address these issues. Compressed sensing employs advanced SP methods to create an image from incomplete K-space samples, allowing for quicker picture generation while avoiding Gibbs ringing. SP experts may work with physicians and technicians to disrupt the medical imaging business using these methods, which are constantly developing. Indeed, the NYU School of Medicine and Facebook's Artificial Intelligence Research (FAIR) division have teamed together to investigate how artificial intelligence might utilize parallel imaging to speed up scan generation.

\section{Exploring the Brain with MRI: Radical New Innovations}

Over the next several decades, neurological illnesses will put an increasing strain on health-care systems, and technology will be required to assist scientists and clinicians better comprehend how these ailments develop and advance. Engineers are using processing steps in new, cutting-edge MRI methods to investigate brain processes that are still a mystery to medical research. Engineers may utilize these methods as they develop to:

- Create "wiring maps" of neuronal synaptic connections to assist surgeons in the excision of brain tumors

- Improve MR spectroscopic, a nonsurgical technique that detects chemical brain changes, including tumor presence.

- Advanced MRI Displacement Imaging techniques are being utilized to research Alzheimer's, Parkinson's, and ADHD, as well as forecast who would benefit from a certain treatment.

- Whenever you tell a falsehood, see which areas of your brain are engaged.

\section{CONCLUSION}

Image processing techniques are critical for understanding and collecting data from these images. Medical imaging is the process of gaining a better understanding of a body's inside sections or interior portion sizes for diagnostic purposes, surgical treatment, and graphical demonstration of the component of a few anatomical structures for diagnostic purposes, medicative intrusion, and graphical demonstration of the component of a few anatomical structures for diagnostic purposes, medicative intrusion, and graphical demonstration of the component of a few anatomical structures. These visual 
manifestations are proficiently processed, analyzed with strategic targets, and released publicly and communicated to multiple locations thanks to improved communication connections and policies and procedures in Medical science, such as Picture Archiving and Communication Systems (PACS), Interactive Image analysis and Communication services. From this research, the following findings may be drawn:

- Elastix and Camino are two possible medical image analysis technologies.

- Another medical image analysis tool included in the platform is Gimias.

A survey report was prepared as part of this study, which included an evaluation of 15 instruments. Finally, this article reviews current medical image analysis technologies and suggests various medical image evaluation techniques for use in medical picture assessment. Only medical imaging tools are included in the scope. Each technology for MIP has its own set of restrictions. As a result, this document may be utilized as a source of information. Nonetheless, this review article has several drawbacks, such as the fact that it only covers 15 MIP technologies. In the following article, suggestions for further work include increasing the number of surveying options.

\section{References}

[1]. W. schOrner, "Picture Archiving and Communication Systems and Videoconference for Medical Communication", Investigative Radiology, vol. 28, pp. S76-78, 1993. Doi: 10.1097/00004424-199308003-00040.

[2]. D. Peruzzo, F. Zanderigo, A. Bertoldo, G. Pillonetto, M. Cosottini and C. Cobelli, "Assessment of clinical data of nonlinear stochastic deconvolution versus block-circulant singular value decomposition for quantitative dynamic susceptibility contrast magnetic resonance imaging", Magnetic Resonance Imaging, vol. 29, no. 7, pp. 927-936, 2011. Doi: 10.1016/j.mri.2011.02.006.

[3]. K. Suzuki, I. Horiba and N. Sugie, "Neural edge enhancer for supervised edge enhancement from noisy images", IEEE Transactions on Pattern Analysis and Machine Intelligence, vol. 25, no. 12, pp. 1582-1596, 2003. Doi: 10.1109/tpami.2003.1251151.

[4]. Weiyang Zhou, "Verification of the nonparametric characteristics of backpropagation neural networks for image classification", IEEE Transactions on Geoscience and Remote Sensing, vol. 37, no. 2, pp. 771-779, 1999. Doi: 10.1109/36.752193.

[5]. S. D, "Metro Water Fraudulent Prediction in Houses Using Convolutional Neural Network and Recurrent Neural Network", Revista Gestão Inovação e Tecnologias, vol. 11, no. 4, pp. 1177-1187, 2021. Doi: 10.47059/revistageintec.v11i4.2177.

[6]. M. Waseem Khan, "A Survey: Image Segmentation Techniques", International Journal of Future Computer and Communication, pp. 89-93, 2014. Doi: 10.7763/ijfcc.2014.v3.274

[7]. T. Cevik, M. Fettahoglu, N. Cevik and S. Yilmaz, "FTSH: a framework for transition from square image processing to hexagonal image processing", Multimedia Tools and Applications, vol. 79, no. 11-12, pp. 7021-7048, 2019. Doi: 10.1007/s11042-019-08487-z.

[8]. B. Avants, N. Tustison, G. Song, P. Cook, A. Klein and J. Gee, "A reproducible evaluation of ANTs similarity metric performance in brain image registration", NeuroImage, vol. 54, no. 3, pp. 2033-2044, 2011. Doi: 10.1016/j.neuroimage.2010.09.025.

[9]. I. Oguz et al., "Minimally interactive placenta segmentation from three-dimensional ultrasound images", Journal of Medical Imaging, vol. 7 , no. 01, p. 1, 2020. Doi: 10.1117/1.jmi.7.1.014004.

[10]. G. Wills, "Visualization toolkit software", Wiley Interdisciplinary Reviews: Computational Statistics, vol. 4, no. 5, pp. 474-481, 2012. Doi: $10.1002 /$ wics. 1224

[11]. A. Haldorai and A. Ramu, "Security and channel noise management in cognitive radio networks," Computers \& Electrical Engineering, vol. 87, p. 106784, Oct. 2020. doi:10.1016/j.compeleceng.2020.106784

[12]. A. Haldorai and A. Ramu, "Canonical Correlation Analysis Based Hyper Basis Feedforward Neural Network Classification for Urban Sustainability," Neural Processing Letters, Aug. 2020. doi:10.1007/s11063-020-10327-3

[13]. D. Devikanniga, A. Ramu, and A. Haldorai, "Efficient Diagnosis of Liver Disease using Support Vector Machine Optimized with Crows Search Algorithm," EAI Endorsed Transactions on Energy Web, p. 164177, Jul. 2018. doi:10.4108/eai.13-7-2018.164177

[14]. H. Anandakumar and K. Umamaheswari, "Supervised machine learning techniques in cognitive radio networks during cooperative spectrum handovers," Cluster Computing, vol. 20, no. 2, pp. 1505-1515, Mar. 2017.

[15]. H. Anandakumar and K. Umamaheswari, "A bio-inspired swarm intelligence technique for social aware cognitive radio handovers," Computers \& Electrical Engineering, vol. 71, pp. 925-937, Oct. 2018. doi:10.1016/j.compeleceng.2017.09.016

[16]. R. Arulmurugan and H. Anandakumar, "Early Detection of Lung Cancer Using Wavelet Feature Descriptor and Feed Forward Back Propagation Neural Networks Classifier," Lecture Notes in Computational Vision and Biomechanics, pp. 103-110, 2018. doi:10.1007/978-3319-71767-8_9 